\title{
Computational and experimental studies on oxalic acid imprinted polymer
}

\author{
KIRAN KUMAR TADI and R V MOTGHARE* \\ Department of Chemistry, Visvesvaraya National Institute of Technology, South Ambazari Road, \\ Nagpur 440 011, India \\ e-mail: rkkawadkar@chm.vnit.ac.in
}

MS received 13 March 2012; revised 30 July 2012; accepted 13 September 2012

\begin{abstract}
Computational approach plays an important role to pre-evaluate the interactions between template and functional monomer, so that to choose functional monomer having stronger interactions with template during synthesis of molecularly imprinted polymers (MIPs). Hence template-monomer interactions in pre-polymerization were mainly focused. In this paper, computational chemistry was applied to screen the number of mol of functional monomer that interacts with one mol of template. Intermolecular interactions between oxalic acid and acrylamide have been investigated. The binding energies $\Delta \mathrm{E}_{\mathrm{bind}}$ were calculated by DFT (B3LYP) level of theory with the $6-31+\mathrm{G}(\mathrm{d}, \mathrm{p})$ basis set. It was found that four mol of acrylamide were sufficient to interact with one mol of oxalic acid in the pre-polymerization mixture. Four possible conformations and frequency calculations were performed to locate minima. Oxalic acid specific bulk polymer was obtained by the thermal initiated free radical co-polymerization of acrylamide and ethylene glycol dimethacrylate with oxalic acid as template and acetonitrile as porogen. The synthesized MIP efficiently adsorbed oxalic acid from aqueous solutions. The binding parameters of MIP and non-imprinted polymer (NIP) were compared by Freundlich and Langmuir adsorption isotherms.
\end{abstract}

Keywords. Computational approach; molecularly imprinted polymer; oxalic acid; adsorption studies.

\section{Introduction}

Molecular imprinting technology is a convenient approach for preparing synthetic receptor that possesses user defined recognition properties (Wulff 1993: Mosbach 1914). It involves, self assembled complexation of a substrate to functional monomers to form a pre-polymer complex, ${ }^{1}$ which is 'locked-in' to place by co-polymerization with an excess of cross linking monomer.

Templates interact specifically with various functional monomers in different way to make covalent, non-covalent or semi-covalent complexes. The noncovalent approach was introduced and further developed by Mosbach et al..$^{2,3}$

Synthesis of MIP involves, dissolving the template and functional monomer of particular ratio in a solvent or porogen to form a pre-polymerization complex, followed by addition of a crosslinker and an initiator. After thermal or UV irradiated polymerization, the template molecule is extracted, forming cavities complementary to the shape and size of the template in the

*For correspondence polymer matrix. Thus, the MIP can selectively adsorb the template molecule from structural analogues of the template. ${ }^{4}$ The control polymer or NIP can be synthesized in the same manner as MIP, but in the absence of template. MIPs are capable of recognizing traces of analytes in bio-fluids like blood, urine, etc. and therefore have been finding great application in different fields such as solid phase extraction (SPE), biomimetic assays, sensors and catalysis. ${ }^{5,6}$

Although, the synthesis of MIP looks quite easy, it is difficult to screen out the best functional monomer having more binding interactions with template. Combinatorial chemistry and molecular modelling are the most promising tools for the development of MIPs with enhanced recognition properties. ${ }^{7}$ But the former suffers from waste of chemicals and time. Modelling of template-functional monomer interactions by computational method is a successful approach towards more rational designing of MIP. ${ }^{8-11}$

In this work, oxalic acid which is widely distributed in various organisms, fungi, plants and animals was selected as template. High levels of oxalic acid remove calcium from blood with severe disturbances in the activity of heart and neural system. It was found that oxalic acid may cause digestive tract irritation, 
kidney damage and therefore there is an increasing demand for its determination in biofluids. ${ }^{12-14}$ Since, oxalic acid is highly hydrophilic $\left[\log \mathrm{K}_{\mathrm{O} / \mathrm{W}}=-0.7\right.$ ] and extensively forms hydrogen bonding with aqueous media, computational methods were adopted to achieve the best monomer-template interactions in prepolymerization mixture. Acrylamide can be used as a functional monomer in polymerization reactions, when the template has hydrogen bonding and acidic functional groups as proved in case of galactose imprinting. ${ }^{15,16}$ After optimization of template: functional monomer ratio, two geometrical isomers of oxalic acid viz., alpha and beta ${ }^{17}$ with two different conformations of each isomer were optimized at DFT (B3LYP) level of theory with 6-31G (d) basis set. The bonding energies $\Delta \mathrm{E}_{\text {Bind }}$ were calculated at B3LYP $/ 6-31+\mathrm{G}(\mathrm{d}, \mathrm{p})$ level of approximation. Furthermore, the hydrogen bonding interactions between acrylamide and oxalic acid were characterized by consideration of atomic charges and IR frequencies.

\section{Materials and methods}

\subsection{Experimental methods}

Acrylamide (ACR), Ethylene glycol dimethacrylate (EGDMA) were purchased from Sigma Aldrich. Acetonitrile and Oxalic acid were procured from Merck. Azobisisobutyronitrile (AIBN) was supplied by Across Organics and re-crystallized from methanol before use. All chemicals were of analytical grade unless mentioned and all solutions are prepared in double distilled water. Standard sieve of BSS number 200 was used to get fine particles.

A Shimazdu model UV-1800 spectrophotometer equipped with quartz cell of $1 \mathrm{~cm}$ path length was used for recording absorbance spectra. Toshniwal $\mathrm{pH}$ meter was used for measuring $\mathrm{pH}$ of the eluents.

\subsection{Synthesis of oxalic acid imprinted polymer and NIP}

Oxalic acid imprinted polymer particles were prepared by taking $0.25 \mathrm{mmol}$ oxalic acid, $1.0 \mathrm{mmol}$ of acrylamide (functional monomer), and $5 \mathrm{ml}$ of acetonitrile in $15 \mathrm{ml}$ of glass vial. The contents were shaked well to ensure formation of pre-polymerization complex. Subsequently, $10 \mathrm{mmol}$ of EGDMA (cross linker) and initiator AIBN $(0.025 \mathrm{~g})$ were added. The mixture was purged with Nitrogen for 10 min to create inert atmosphere. The vial was completely sealed and kept in water bath at $55^{\circ} \mathrm{C}$ for $12 \mathrm{~h}$ to complete polymerization reaction. The control or non-imprinted polymer was also prepared in the same manner but in the absence of oxalic acid. The glass vial was broken to get the polymer, washed with methanol to remove unreacted precursors and dried. Then it was ground using mortar - pestle and sieved with a standard sieve of size 75 microns to get fine particles.

The fine particles of polymer were washed with double distilled water to elute the template and then complete elution of template was confirmed from the $\mathrm{pH}$ of the eluent as well as by UV/VIS spectrophotometry. The particles were dried under vacuum at $50^{\circ} \mathrm{C}$ overnight and then used for further studies. Figure 1 shows a schematic representation of molecular imprinting step including polymer synthesis and template removal.

\subsection{Binding studies}

The binding capacity of MIP and NIP were determined by batch rebinding experiments. In the binding assay,
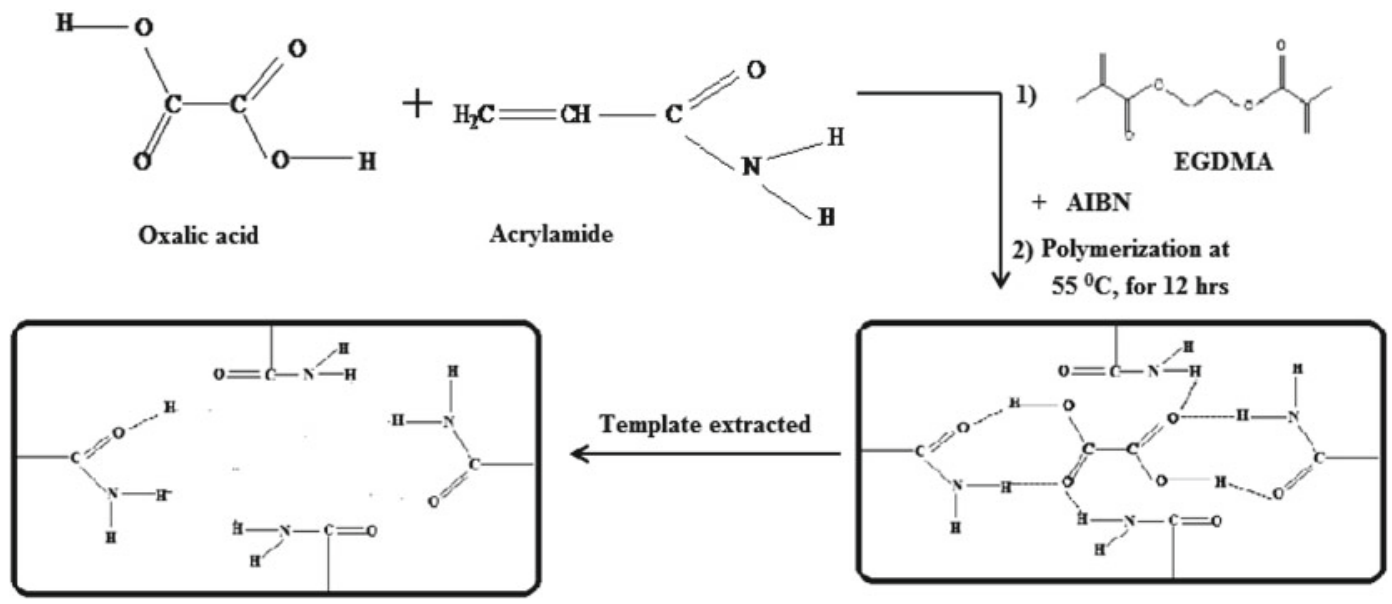

Figure 1. Schematic representation for the synthesis of oxalic acid imprinted polymer. 
$0.1 \mathrm{~g}$ of MIP or NIP was added to the $20 \mathrm{ml}$ aqueous solution of the analyte of different concentrations and stirred for $5 \mathrm{~h}$ at $25^{\circ} \mathrm{C}$. The polymer particles were filtered off and the filtrate was analysed for oxalic acid by UV/VIS spectrophotometer. The quantity of the bound analyte was determined by the subtracting the equilibrium amount of template from initial quantity of template. The experimental binding data were fitted to the Freundlich and Langmuir adsorption models.

\section{Computational methods}

\subsection{Geometry optimization and energy calculations}

The three-dimensional structure of the monomer, template and monomer-template complexes were built up with the aid of AVOGADRO programme ${ }^{18}$ and preoptimized by $\mathrm{MMFF}^{19}$ force field applying its implementation in the Avogadro programme. ${ }^{20}$

The molecular geometries were optimized by using density functional theory at the B3LYP/6-31+G(d,p)// B3LYP/6-31G(d) level ${ }^{21,22}$ implemented in Gaussian $03 .{ }^{23}$

The optimizations were followed by computations of the harmonic vibrational frequencies, so that to check local minima and no imaginary frequencies were obtained. Thus, the geometry and vibrational frequencies of different possible forms $\alpha$-OA-ACR and $\beta$-OA-ACR for the hydrogen bond interactions among $\alpha$ - and $\beta$ - oxalic acid with acrylamide have been fully optimized at B3LYP/6-31G(d) level of approximation.

The single point energies of each optimized conformations were calculated by using B3LYP $/ 6-31+\mathrm{G}(\mathrm{d}, \mathrm{p})$ basis set. The minimum binding energies of one mol of template: varying mol of functional monomer (1: $\mathrm{N}$ ratio) are listed in table 1 . The $\Delta \mathrm{E}$ values were calculated by using following equation.

$$
\begin{aligned}
\Delta \mathrm{E}= & \mathrm{E}(\text { template }- \text { monomer })-\mathrm{E}(\text { template }) \\
& -\Sigma \mathrm{E}(\text { monomer })
\end{aligned}
$$

The basis set superposition error (BSSE) which often substantially affects the calculated stabilization energies was corrected by means of the counterpoise method. ${ }^{24,25}$

\section{Results and discussions}

\subsection{Selection of proper functional monomer}

Selection of suitable functional monomer plays an important role in deciding binding capacity of MIPs. Oxalic acid is a highly hydrophilic compound containing two carboxylic acidic groups can form six hydrogen bonds. Acrylamide was chosen as functional monomer which shows strong interactions with a molecule having hydrogen bonding groups viz., acidic functional groups. ${ }^{15}$ It gives good recognition properties especially in polar solvents.

\subsection{Optimization of template: functional monomer ratio}

If excess mol of functional monomer are used, only specific number of mol reacts with template and rest which remain as excess leads to non-specific binding ${ }^{26}$ and hence decreases the binding capacity. So, first the number of moles of functional monomer that interacts with one mol of template was optimized. From the results (table 1), it can be concluded that order of the molecular interaction is as follows: OA-AA $(1: 4)>\mathrm{OA}-\mathrm{AA}(1: 3)$ $>$ OA-AA (1:2) > OA-AA (1:1). These results show the complex in 1:4 ratio has highest binding energy and thus leads to the formation of the most stable complex.

After optimization of template: monomer ratio, four possible forms (cis-OA-AA (1-2) and trans-OA-AA (1-2)) were optimized to know the most stable conformation. It could be observed from table 2 that strong interactions were found between trans-oxalic acid and acrylamide (2) with $\Delta \mathrm{E}_{\text {Bind }}-46.74 \mathrm{kcal} / \mathrm{mol}$.

\begin{tabular}{|c|c|c|}
\hline Molecules & Energy(Hartrees/particle) & Interaction energy ${ }^{\mathrm{a}} \Delta \mathrm{E}(\mathrm{kcal} / \mathrm{mol})$ \\
\hline Oxalic acid & -378.3508 & --- \\
\hline Acrylamide(AA) & -247.3193 & --- \\
\hline $\mathrm{OA}-\mathrm{AA}$ & -625.69296 & $-14.31(0.8283)$ \\
\hline $\mathrm{OA}-(\mathrm{AA})_{2}$ & -873.02680 & $-23.12(1.5173)$ \\
\hline $\mathrm{OA}-(\mathrm{AA})_{3}$ & 1120.36518 & $-35.33(6.2750)$ \\
\hline $\mathrm{OA}-(\mathrm{AA})_{4}$ & -1367.69308 & $-40.81(3.7085)$ \\
\hline
\end{tabular}

Table 1. Binding energies between template and the monomer(s).

${ }^{\mathrm{a}}$ In parentheses the corresponding values of BSSE correction for the interaction energy are shown 
Table 2. Binding energies $(\Delta \mathrm{E})$ for $c i s$ oxalic acid-AA(1-2) and trans oxalic acid$\mathrm{AA}(1-2)$.

\begin{tabular}{lcc}
\hline Compound & Energy(Hartrees/particle) & Interaction energy $^{\mathrm{a}} \Delta \mathrm{E}(\mathrm{kcal} / \mathrm{mol})$ \\
\hline Cis-OA-AA (1) & -1367.68883 & $-38.14(2.5194)$ \\
Cis-OA-AA (2) & -1367.69053 & $-39.206(2.8802)$ \\
Trans-OA-AA (1) & -1367.68183 & $-33.747(2.8802)$ \\
Trans-OA-AA (2) & -1367.70256 & $-46.749(3.4262)$
\end{tabular}

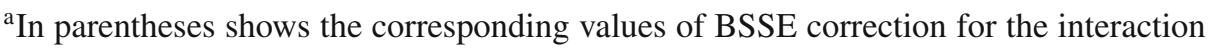
energy

In non-covalent approach, even though the functional monomer-template interact through ionic-bonds, hydrogen bonding, dipole-dipole etc., hydrogen bonding plays an important role in stabilizing the prepolymerization complex. ${ }^{27,28}$ The structure of all the four studied complexes for 1:4 ratio are shown in figure 2. It is evident from the structures that cis-OAAA(1-2) interacts with three acrylamide molecules and one remains free but trans-OA-AA(2) interacts with four functional monomer molecules. Trans-OA-AA(1) shows hydrogen bonding with two functional monomer molecules and the other two molecules has weaker interactions between $\mathrm{C}=\mathrm{O}$ of $\mathrm{OA}$ and $\mathrm{N}-\mathrm{H}$ of $\mathrm{AA}$ (distance between $\mathrm{H}_{35^{---}} \mathrm{O}_{4}$ is $2.06 \AA$ and for $\mathrm{H}_{42^{---}}$ $\mathrm{O}_{3}$ is $2.09 \AA$ ), which indicates that four molecules of acrylamide interacts with trans-OA(1).

4.2a Atomic charges: Table 3 summarizes atomic charges in $\mathrm{OA}$ and AA. To avoid presenting a large amount of data, only those atoms exhibiting significant changes in their atomic charges after complexation are
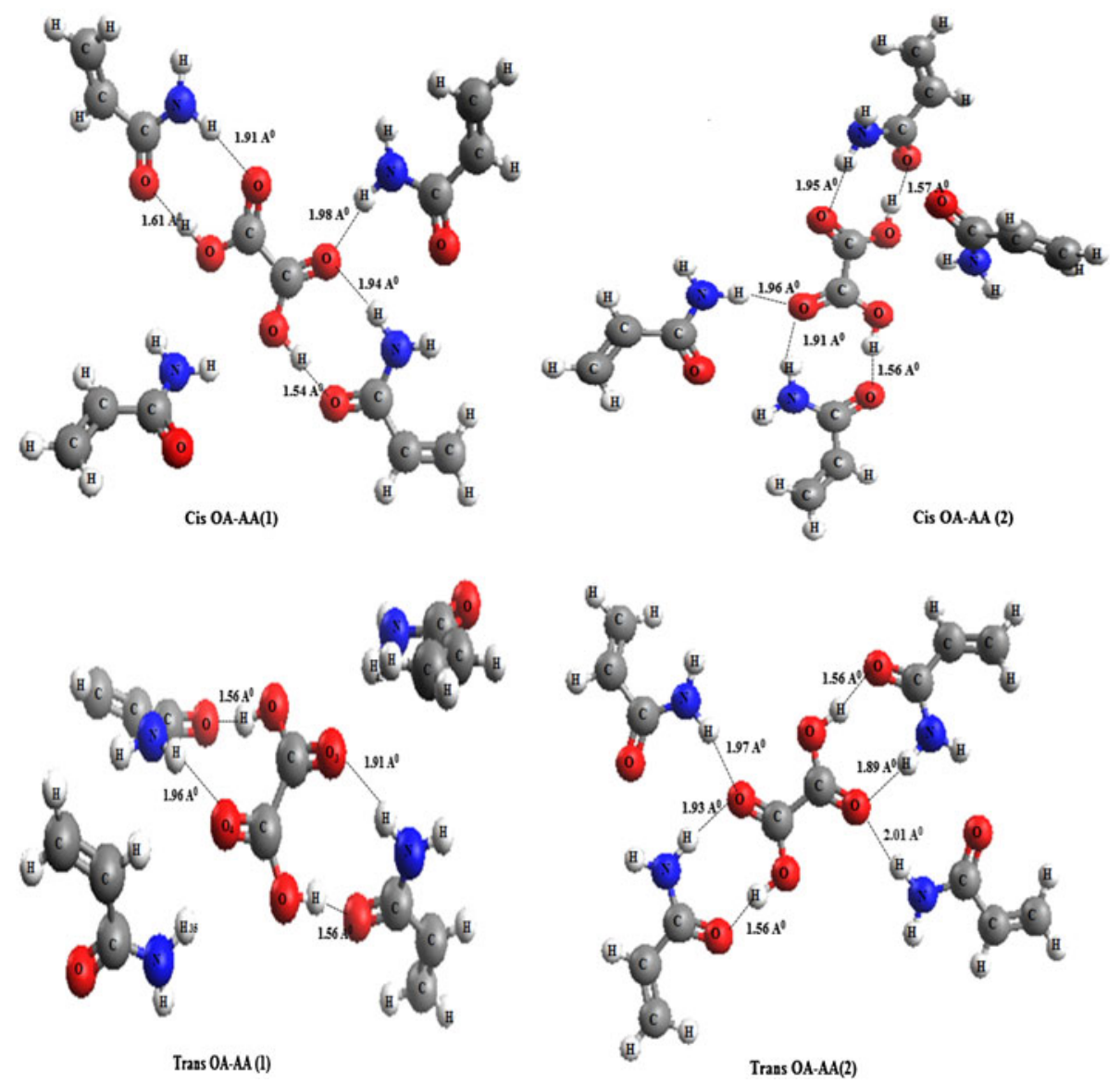

Figure 2. Optimized structures showing hydrogen bonding for the four complexes, cis-OA-AA (1-2) and trans-OA-AA (1-2) ( $)$. 
Table 3. Atomic charges in OA and AA before and after complex formation.

\begin{tabular}{lccccr}
\hline \multirow{2}{*}{ Molecule } & & & \multicolumn{3}{c}{ Atomic charges } \\
\cline { 4 - 6 } Oxalic acid & Atom number & Atom & Individual & Complex(1:1) & Complex (1:4) \\
& 3 & $\mathrm{O}$ & -0.4104 & -0.4828 & -0.5871 \\
& 4 & $\mathrm{O}$ & -0.4104 & -0.4014 & -0.6269 \\
AA & 7 & $\mathrm{H}$ & 0.3792 & 0.3848 & 0.4828 \\
& 8 & $\mathrm{H}$ & 0.3792 & 0.4004 & 0.4912 \\
& 28 & $\mathrm{O}$ & -0.5538 & -0.6247 & -0.6337 \\
& 29 & $\mathrm{O}$ & -0.5538 & --- & -0.5956 \\
& 33 & $\mathrm{H}$ & 0.2941 & 0.3095 & 0.4039 \\
& 36 & $\mathrm{H}$ & --- & --- & 0.3999 \\
& 38 & $\mathrm{H}$ & --- & --- & 0.3935 \\
& 41 & $\mathrm{H}$ & --- & -- & 0.3712 \\
\hline
\end{tabular}

shown in table 3 . The atomic charges participating in complex formation changed more significantly in comparison with those of other atoms. From these changes, it was deduced that atoms of OA and AA are participating in complex formation. Furthermore, to select most stable complex, changes in the atomic charges before interacting and after complex formation were studied. As the interaction between monomer and template become stronger, the change in atomic charges increased. It is obvious that charge variation after complex formation is more significant in (1:4) OA-AA than in $(1: 1)$ OA-AA.

4.2b Simulated IR spectra: The simulated infrared spectra computed at B3LYP/6-31G(d) for (1:4) transOA-AA-2 and monomeric units are given for comparison (see supplementary information figure S1). The most important change observed in the infrared spectra upon 1:4 complex formation is crucial red shift and this can be result of $v(\mathrm{~N}-\mathrm{H})$ stretching of the four monomeric units of acrylamide at $2640 \mathrm{~cm}^{-1}$. The adsorption due to the hydrogen bonded at $\mathrm{O}-\mathrm{H}$ stretch at $3421 \mathrm{~cm}^{-1}$ and a broad peak at $1650 \mathrm{~cm}^{-1}$ due to hydrogen bonded $\mathrm{C}=\mathrm{O}$ group in oxalic acid can be observed. These adsorptions show red shift denoting strong interactions between the four molecules of acrylamide and one molecule of oxalic acid (trans-OAAA-2) complex. The IR spectra of anhydrous oxalic acid and trichloro acetic acid were studied by Adams and Kims. ${ }^{29}$

\subsection{Batch rebinding experiments}

Two binding models (Freundlich and Langmuir adsorption models) were applied to study the binding characteristics of MIP and NIP. 4.3a Freundlich adsorption isotherm: It is the most commonly applied continuous distribution model. The FI is a power function (Eq. (1)).

$$
\text { i.e., } \mathrm{B}=\mathrm{aF}^{\mathrm{m}} \text {, }
$$

where the pre-exponential factor ' $\mathrm{a}$ ' is measure of the capacity $\left(\mathrm{N}_{\mathrm{t}}\right)$ and average affinity $\left(\mathrm{K}_{\mathrm{o}}\right)$ and $\mathrm{m}$ is the heterogeneity index. The heterogeneity is inversely proportional to $\mathrm{m}$. The Freundlich equation can be rearranged to a linear form

$$
\log \mathrm{B}=\mathrm{m} \log \mathrm{F}+\log \mathrm{a} .
$$

By plotting $\log \mathrm{B}$ versus $\log \mathrm{F}$ (see supplementary information figure S2), the values of ' $m$ ' and ' $a$ ' can be calculated from the slope and y-intercept respectively.

4.3b Langmuir adsorption isotherm: The Langmuir isotherm (Eq. 3) is a discrete model that involves only one type of binding site:

$$
\mathrm{B}=\frac{\mathrm{NKC}}{1+\mathrm{KC}},
$$

where $\mathrm{K}$ is a adsorption constant and $\mathrm{N}$ is the total6 number of binding sites. The Langmuir isotherm equation (4) can be easily transformed into a linear form as follows.

$$
\frac{1}{B}=\frac{1}{N K C}+\frac{1}{N}
$$

The plot between 1/B versus 1/C for the oxalic acid imprinted polymer is shown in supplementary information (figure S3).

The binding isotherms were fitted to the two models and the results are reported in supplementary information table $\mathrm{S} 1$. 


\section{Conclusions}

Quantum chemical calculation at B3LYP level of theory with the $6-31 \mathrm{G}^{*}$ basis set were applied for the optimization of oxalic acid: acrylamide mol ratio in the pre-polymerization process. It was found that 1:4 template monomer ratio is the best configuration. The hydrogen bonding interactions between acrylamide as monomer and cis-, trans-oxalic acid as templates in MIP preparation were studied. More favoured interactions were found for the trans-oxalic acid form, in which carboxylic acid groups of one carbon are oriented in the same side of amide group of monomer. The trans-OA-AA-2 complex is the most stabilized complex with binding energy of $-46.749 \mathrm{kcal} / \mathrm{mol}$, which are corrected by taking into account the basis set super station error. From these results, oxalic acid MIP with 1:4 oxalic acid: acrylamide mole ratio was synthesized by bulk polymerization method. Batch rebinding experiments were performed. The maximum adsorption capacity, equilibrium constant and binding affinity were obtained by Langmuir and Freundlich adsorption isotherm models. The synthesized MIP exhibits specific molecular recognition ability towards the template molecule.

\section{Acknowledgements}

We thank the Electronics and Communication Engineering Department, Visvesvaraya National Institute of Technology (VNIT), Nagpur for giving opportunity to work on Gaussian Software.

\section{References}

1. Yan M and Ramstrom O 2005 Molecularly imprinted materials, (New York: Marcel Dekker) pp 25

2. Vlatakis G, Andersson L I, Muller R and Mosbach K 1993 Nature 361645

3. Andersson LI, Muller R, Vlatakis G and Mosbach K 1995 Proc. Natl. Acad. Sci. USA 924788

4. Yan H and Row K H 2006 Int. J. Mol. Sci. 7155
5. Piletsky S A, Alcok S and Turner A P F 2001 Trends Biotechnol. 199

6. Mahony J O, Nolan K, Smyth M R and Mizaikoff B 2005 Anal. Chim. Acta 534(1) 31

7. Nostrum C F V 2005 Drug Discov. Today 1119

8. Chianella I, Piletsky S A, Tothill I E, Chen B and Turner A P F 2003 Biosens. Bioelectron. 18119

9. Khan M S, prateek S, Wate and Krupadam R J 2011 J. Mol. Model. 18(5) 1969

10. Nicholls I A, Hakan S A, Christy C, Henning H, Björn C G K, Jesper G. Karlsson, Mahony J O, Annika M R, Johan R K, and Susanne W 2009 Biosens. Bioelectron. 25543

11. Siavash R, Edris-Tabrizi F, Mehran J, Mohammad R G and Parviz N 2009 J. Mol. Model. 15829

12. Health Council of the Netherlands: Committee on Updating of Occupational Exposure Limits. Oxalic acid; Health-based Reassessment of Administrative Occupational Exposure Limits. The Hague: Health Council of the Netherlands, 2004;2000/15OSH/106

13. Menache R 1974 Clin. Chem. 201444

14. Powers H H and Levatin P 1944 J. Biol. Chem. 154207

15. Yu C and Mosbach K 1997 J. Org. Chem. 624057

16. Okutucu B, Onnal S and Telefoncu A 2009 Talanta 78 1190

17. Chitra R, Amit Das, Choudhury R R, Ramanadham M and Chidambaram R 2004 Pramana - J. Phys. 63263

18. http://avogadro.openmolecules.net/

19. Halgren T A and Merck 1996 J. Comp. Chem. 17490

20. Roberta D S, Mari R L, Mario A, Fabio D S, Donato C and Giuseppe V 2009 Molecules 142632

21. Yang W and Wu Q 2002 Phys. Rev. Lett. 89 143002/1

22. Parr R G and Yang W 1995 Annu. Rev. Phys. Chem. 46 701

23. Gaussian 03, Revision C.02, Gaussian, Inc.: Wallingford, CT, USA, 2004

24. Schwenke D W and Truhlar D G 1985 J. Chem. Phys. 822418

25. Michael J F, Janet E D B, Stephen B J and Henry F S 1986 J. Chem. Phys. 842279

26. Komiyama M, Takeuchi T, Mukawa $\mathrm{T}$ and Asanuma H 2003 Molecular imprinting: from fundamentals to applications (Wiley: VCH) pp 67

27. Piletsky S A, Karim K, Piletska E V, Day C J, Freebairn K W, Legge C and Turne A P F 2001 Analyst 1261826

28. Karim K, Florent B, Regis R, Elena V P, Antonio G, Chianell I and Piletsky S A 2005 Adv. Drug Deliver Rev. 571795

29. Adams J and Kims H 1973 Spectrochimica Acta 29(4) 675 